\title{
The control system of the yeast drying process
}

\author{
Piotr Wolszczak ${ }^{1, a}$ and Waldemar Samociuk ${ }^{2, b}$ \\ ${ }^{1}$ Lublin University of Technology, Faculty of Mechanical Engineering, Nadbystrzycka 36, Lublin 20-618, Poland \\ ${ }^{2}$ University of Life Sciences in Lublin, Faculty of Production Engineering, 28 Głęboka Street, Lublin 20-612, Poland
}

\begin{abstract}
The article presents the results of choosing how to control a real non-linear object. Yeast drying requires a precise temperature control due to the possibility of overheating. The object changes properties during of the process flow. Object identification is used and a mathematical model is developed. The model is used to select roboust control methods. The results are compared to the system of two PID regulators used in practice.
\end{abstract}

\section{Introduction}

When designing control systems, there is an inevitable compromise between the complexity of the controlled process model and the effort necessary to obtain a reliable mathematical description. The design methods can be broadly divided into two main classes: the indirect class (model-based approach) and the direct class (model-free approach) [1]. Finding models from the laws of physics can be difficult and time-consuming. Therefore control systems are designed based on experimental data. Indirect approaches use the collected data to identify a controlled process model. This method is based on the system identification tools [2-8]. The mathematical model (identifeid with data or derived from physical laws) is used to synthesize the controller in accordance with the design goals. On the other hand, the direct approach is not aimed at obtaining a precise model of the controlled process. In direct approaches, the identification step is limited to the basic process properties (such as static characteristics, time constant and delay). These properties are used to determine the structure of the control system (controller type) and the settings of this controller.

The reasons for the uncertainty of the models of object can be: using linearization to describe non-linear objects approximate or variable model parameters, errors of the identification based on measurement data, no description of parameters in the range of higher sampling rates, changing the object working conditions.

It is particularly difficult to design control systems for processes in which the model parameters change their values. In such situations, it must be taken into account the stability of the control system during these changes. In the literature for such systems there is a

\footnotetext{
ae-mail: p.wolszczak@pollub.pl

be-mail: waldemar.samociuk@up.lublin.pl
}

term of robust control which takes into account the object model (Plant) [9, 10]. The robust control system is designed so that the controller operates correctly in conditions when certain parameters of the object are uncertain (imprecisely determined or will change in a limited range).

Robust control requires a priori information about the range of uncertain or limits of properties variability and ensures that if the changes are within limits, the change of the adopted process control principle is no necessary.

\subsection{Internal Model Control - Principle of operation}

Internal Model Control (IMC) is a control that uses an internal process model. Assuming that the controller is an exact approximation of the real process, the output of the system will always be equal to the set value. However, designing a controller that is the exact opposite of the process is not always realizable. The use of a feedback loop is necessary if the process model is inaccurate or incomplete. A loopback system eliminates the effects of an inaccurate model to a certain extent and increases the system's robustness to interference and noise affecting the control object.

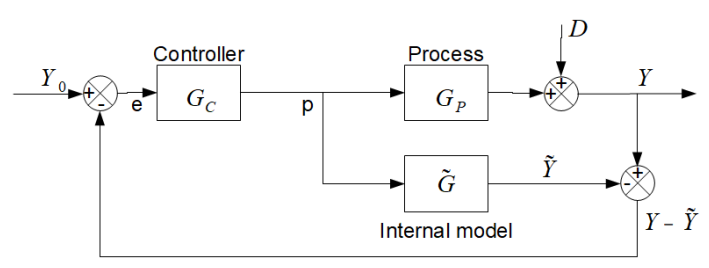

Figure 1. Internal Model Control (IMC) Diagram. $Y_{0}$ - sepoint signal, e - error signal, p - control signal, D - interference, $\tilde{Y}$ output of the internal model, Y - output signal. 


\section{Yeast drying}

Drying yeast has an adverse effect on their fermentation capacity. The level of degradation depends on the method and parameters of the drying process [11]. During drying, cellular stress occurs [12]. Figure 2 presents offset of the drying surface during drying process of organic material. This offset has an effect on changing the surface of the drying surface. Additionally, lowering the surface of the material influences the change in moisture transport speed. The value of temperature and the rate of its change affect the viability of dried yeast [13, 14]. During industrial production are used various types (strains) of yeast, that have varying degrees of resistant to temperature $[15,16]$. It is possible to use additives (skim milk) to immunize yeast for high temperature [17].

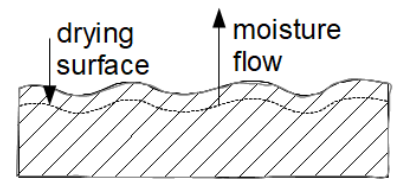

Figure 2. The offset of the drying surface during drying process of organic material.

Drying yeast is an industrial process, non-stationary and non-linear. For such processes the control is difficult. The selection of fixed parameters of the controller of the classical control system for the entire drying cycle is impossible, therefore it is necessary to use complex control systems [18]. For the sake of process safety, taking into account primarily material losses, it is advisable to use security systems and analyze the risk of failure [19, 20].

Figure 3 presents a scheme of a dryer equipped with one heater located outside the drying chamber, which operates without air recirculation. The air used for drying is mixed from two air streams. Thermodynamic transformations accompanying this process are shown in Figs. 4. The drying process in the adiabatic process is different than in the real case. The figure shows the heat loss index $Q_{\text {loss }}$ to the dry air mass flow $L$. The value of $\Delta i_{a d}$ means the difference between enthalpy of humid and atmospheric air.

\section{Subject of research}

The object of the research was the process of industrial yeast drying. The layer of wet yeast is placed on a sieve through which heated air flows. The air temperature is obtained by mixing streams of cool (constant temoerature) and heated air. The control signal is the degree of opening of the hot air throttle. The temperature of the yeast is monitored and controlled during the drying cycle. The drying cycle takes about 450 minutes. During the drying cycle, the temperature of the yeast layer is gradually raised. The yeast temperature control is carried out according to the defined drying curve shown in figure 5. Yeast temperature can not exceed 50 degrees, which would destroy them.

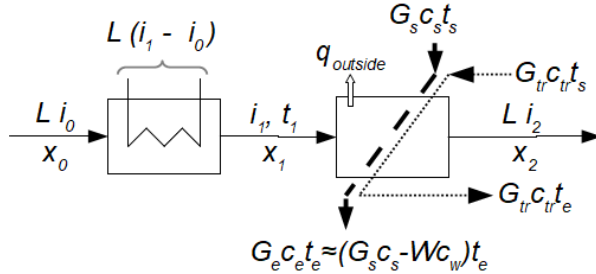

Figure 3. Block diagram of the continuous drying process (normal variant), a), b), c) and d) - heat streams flowing into the dryer, e), f), g) and h) - heat streams flowing out of the dryer; heat balance: $\mathrm{a}+\mathrm{b}+\mathrm{c}+\mathrm{d}=\mathrm{e}+\mathrm{f}+\mathrm{g}+\mathrm{h}$; left block - preheater, right block - drying chamber. $L$ - mass flow of dry air $[\mathrm{kg} / \mathrm{s}], G$ - mass flow of moist material $[\mathrm{kg} / \mathrm{s}], c$ - specific heat $[\mathrm{kJ} /(\mathrm{kg} * \mathrm{~K})], i$ specific enthalpy $[\mathrm{KJ} / \mathrm{kg}]$ of dry air, $x$ - absolute humidity of air [kg of steam $/ \mathrm{kg}$ of dry air], $t$ - temperature [deg $C], W$ - mass flow of evaporated water from the material $[\mathrm{kg} / \mathrm{s}]$; indexes: $s$ - initial value (start), $w$ - water, $e$ - finish value (end), $t r$ - transporter, 0 atmospheric air, 1 - heated air, 2 - humidified air. Based on [21]

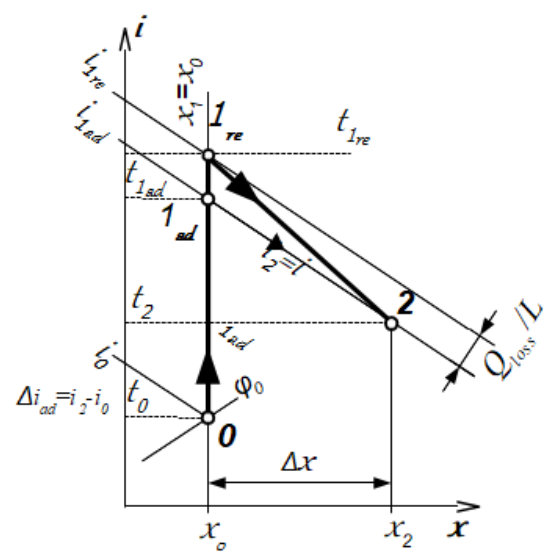

Figure 4. Simplified enthalpy diagram with thermodynamic curves of the drying air in a dryer (norrnal variant), $0-1_{a} d$ heating in the adiabatic process, $0-1_{r} e$ - heating in the real process, $1_{a} d-2$ - adiabatic dampening, $1_{r} e-2$ - real dampening. $i$ - specific enthalpy $[\mathrm{KJ} / \mathrm{kg}]$ of dry air, $x$ - absolute humidity of air $\left[\mathrm{kg}\right.$ of steam $/ \mathrm{kg}$ of dry air], $t$ - temperature $[\operatorname{deg} C] \phi_{0}$ - relative humidity of atmospheric air; indexes: 0 - atmospheric air, 1 - heated air, 2 - humidified air, $a d$ - adiabatic process (theoretic), re - real process (with losses). Based on [21]

Figure 5 presents ten series of drying cycles that contains the following signals: setpoint signal, throttle opening, temperature under the sieve (temperature of the mixture of cool and heated air) and temperature of the yeast layer. The figure shows a block diagram of a closed-loop control system containing the mentioned elements.

During the drying process a change in the physical properties of the object is observed. This change is revealed in the form of increasing the value of the error (the difference between the expected value and the adjusted value). Changing the physical properties of the object also involves changing the sensitivity of the object to changes in the control signal, which increases the risk of 
overheating the yeast.

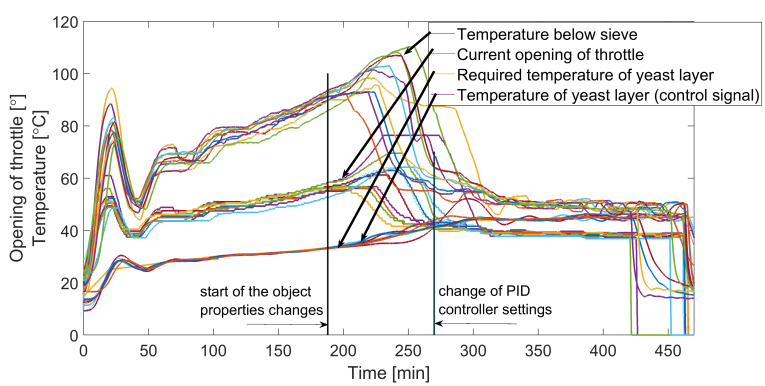

Figure 5. Example sets of signals aquisiited during drying process. Signals: setpoint trace - required temperature of yeast layer on sieve $\left[{ }^{\circ} \mathrm{C}\right]$, control signal - hot air throttle opening $\left[{ }^{\circ}\right]$, the controlled variables - temperature below sieve and yeast layer temperature $\left[{ }^{\circ} \mathrm{C}\right]$.

A typical way to solve this problem is to change the controller's settings during the process. The control system defines the time of switching the controller's operation with the first set to the second set of parameters. One of the problems of such a solution is maladjustment to the moment of real change of the object's property and its character. Changing the settings of the regulator is introduced by step function, while the change in properties can be gradual. One of the ways to solve the problems could be the extension and smoothing of the drying curve. This solution does not ensure full security of the object, and additionally extends the duration of the process and increases its cost.

\section{Methods and results}

The procedure for developing control using the classical PID controlled and IMC system consisted of: registration of the signals from the real object, identification of the mathematical model of the object, (estimated by matching of the output signals) and preparing the systems simulation. During the identification of the model, the order and coefficients of the objects transform functions are adjusted. For every cycle (signals set) has been prepared a set of transform functions with different order value of characteristic equation. The estimation of nonlinear function was also conducted to characterize the object. Identification of the mathematical model was based on the output signals in the entire drying cycle (symbol $\mathrm{AB}$ ) and separately for the first (A) and a second (B) parts. Moreover, due to the use of a temperature signal below the sieve, it was possible to identify the properties of the yeast layer.

Figure 5 presents a block diagram of a closed control system for the yeast drying process. The preparation of a block diagram of the process of drying yeast and the identification of mathematical models of system objects is also helpful in analysis and improving the currently operating control system. The model was used to optimize the previous PID controller settings and the time of switching the
PID controller settings. Based on the observations of the raw signal curves in figure 5, it can be seen that fluctuations in the input and control signals occurs long before the switching of the PID controller settings. The figure 6 presents results of the system simulation in which the changeover time of the controller's settings was corrected. Such readjustment should protect the product from overheating and the controller output signal from saturation (what can be observed in figure 5). The object is descrbed by two transfer function $G_{A}, G_{B}$ switched in 180th time unit of simulation, presented below with approximated values:

$$
\begin{gathered}
G_{A}=\frac{34 s^{3}+2.5 s^{2}+0.001234 s}{s^{4}+33.4 s^{3}+2.466 s^{2}+0.002334 s}, \\
G_{B}=\frac{24.93 s^{3}+0.1079 s^{2}}{s^{4}+25.09 s^{3}+0.1072 s^{2}} .
\end{gathered}
$$

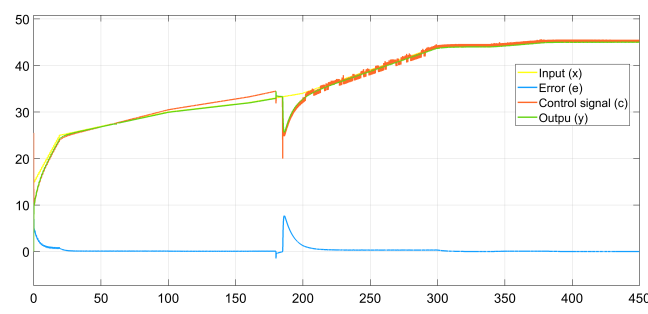

Figure 6. Simulation of drying process characterized by two transfer function $G_{A}, G_{B}$, and two controllers PI $(\mathrm{Kp}=1.7, \mathrm{Ki}=$ $0.785)$ and $\operatorname{PID}(\mathrm{Kp}=1.3, \mathrm{Ki}=0.312, \mathrm{Kd}=0.758)$, witch limited output in range from 20 to $80\left[^{\circ}\right]$ switched in 180th time unit of simulation.

Controlling the process changing its properties using one PID controller proved to be ineffective. To control the process of yeast drying throughout the cycle, two sets of parameters were prepared using a simple sliding-mode model. In order to improve the control quality and product protection from overheating, the robust method was used. The roustness controller was prepared using a mathematical model of the object, wherein the model was identified from signals recorded from the real object in whole drying cycle.The uncertainty of the characteristic equation coefficients in the range of $+/-20 \%$ of nominal values was used. Figure 7 presents a comparison of the step response of PI control systems 1 in the first part of the drying cycle (A), controller 2 (PID) controlling the process in the second part of the drying cycle (B) and the robust controller controlling the process in whole cycle (object transmittance identified on the basis of the signals in both parts of the process, parts $\mathrm{A}$ and $\mathrm{B}$ ). However, the use of the controller 1 for controlling the part B and the controller No. 2 for controlling the model determined on the basis of part A causes instability of both systems. In figure 8 shows the results of the system sensitivity evaluation. Feedback of the inverted model was used

$$
G_{S}=1 / G_{\text {model }} .
$$

The use of a robustness regulator allows the use of one set of settings throughout the entire drying cycle. In this 
way, fluctuations in the output signal caused by a sudden change of the object's properties and a step change in the control parameters were eliminated.

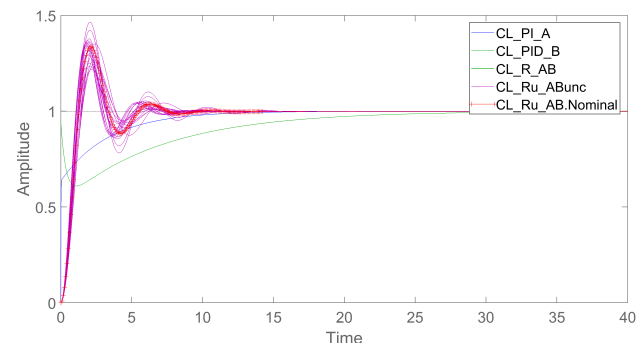

Figure 7. Step response for mathematical models of feedback systems consists of controllers and transfer function of objects: CL_PI_A - PI controller and transfer function of part A, CL_PID_B - PID controller and transfer function of part B, CL_R_AB - Robust controller and transfer function of parts $\mathrm{A}+\mathrm{B}$.

a)
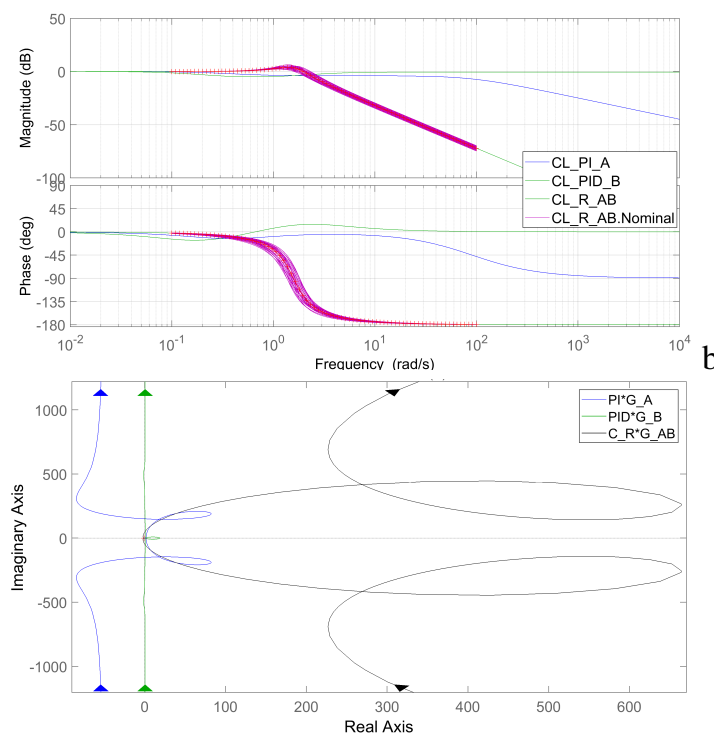

Figure 8. Sensitivity analysis a) Bode plots for closed-loop systems and b) Nyquist plot for mathematical models of open-loop systems consists of controllers and transfer function of objects: $P I * G_{A}$ - PI controller and transfer function of part A, $P I D * G_{B}-$ PID controller and transfer function of part B, $C_{R} * G_{A} B$ - Robust controller and transfer function of parts $\mathrm{A}+\mathrm{B}$.

\section{Conclusions}

A change in the properties of yeasts during drying causes a non-linear response of the temperature signal. This change occurs so quickly that fluctuations in the yeast temperature signal pose a threat to them. The use of robustness controller eliminates this risk and improves quality control. A system with a robust controller reduces the time of adjustment, which is important in the case of sudden changes in the properties of the object. Use of robust controller for non-linear process control is known in the literature, but rarely used in practice. Therefore, it is advisable to test the robustness methodology in industrial conditions.

\section{References}

[1] Battistelli G. et. all., International Journal of Robust and Nonlinear Control 28,3694-3712 (2017)

[2] Hjalmarsson H., Automatica 41, 393-438 (2005)

[3] Wolszczak P., Łygas K., Litak G. Mechanical Systems and Signal Processing 107, 43-52 (2018)

[4] Cechowicz R., Staczek P., Maintenance and reliability 16(2), 198-202 (2014)

[5] Martynenko A., Food Engineering Reviews 9 91-111 (2017)

[6] Yang Li-Ping, Ding Shun-Liang, Litak Grzegorz, Song En-Zhe, Ma Xiu-Zhen, Chaos 25 013105:1-13 (2015)

[7] Harris P., Arafa M., Litak G., Bowen C. R., Iwaniec J., European Physical Journal B 90, 1-11 (2017)

[8] Litak G., Rusinek R., 4th IEEE International Conference on Nonlinear Science and Complexity (NSC) (IEEE, Budapest, Hungary, 2012) 41-44

[9] Garcia C. E., Morari M. Industrial \& Engineering Chemistry Process Design and Development 21, 308323 (1982)

[10] Garcia C. E.Morari M. Industrial \& Engineering Chemistry Process Design and Development 24, 4724841985

[11] Kamińska-Dwórznicka A., Skoniecka A., Advances of Agricultural Sciences Problem Issues (in polish) 573, 35-42 (2013)

[12] Gamero-Sandemetrio E., Payá-Tormo L., GómezPastor R., Aranda A., Matallana E., Microbial Cell (Graz, Austria) 5(4), 184-197 (2018)

[13] Gervais P., Maranon I., Biochemica et Biophysica Acta 1235, 1, 52-56 (1995)

[14] Mensonides FI., Brul S. , Hellingwerf KJ. , Bakker BM. , Teixeira de Mattos MJ., The FEBS Journal 281(3), 825-841 (2014)

[15] Charoensopharat K., Thanonkeo P., Thanonkeo S., Yamada M., Antonie van Leeuwenhoek 108(1), 173190 (2015)

[16] Techaparin A., Thanonkeo P., Klanrit P., Brazilian Journal of Microbiology 48(3), 461-475 (2017)

[17] Lee SB., Choi WS., Jo HJ., Yeo SH., Park HD., AMB Express 6(1), 105 (2016)

[18] Muhammad D., Ahmad Z. Aziz N., Proc. of the 5th International Symposium on Design, Operation and Control of Chemical Processes (PSE Asia, Singapore, 2010) $812-821$

[19] Samociuk W. at all, Przemysł Chemiczny, 95(5), 1000 - 1003 (2016)

[20] Samociuk W., Wyciszkiewicz A., Gołacki K., Otto T., Przemysł Chemiczny, 96(8), 1763-1766 (2017)

[21] Ryniecki A., Convection drying continuous: the exercise of design basics (in polish) (University of Life Sciences, Poznan, Poland) 3-11 